Note

\section{Two Biphenyl Compounds from Rhaphiolepsis umbellata as Its Phytoalexin}

\author{
Keisuke Watanabe, Siti Muslimah \\ Widyastuti* and Fukuji NonaKA* \\ Agricultural Science Research Laboratory, \\ Takarazuka Research Center, Takatsukasa 4-2-1, \\ Takarazuka, Hyogo 665, Japan \\ * Faculty of Agriculture, Saga University. \\ Honjo 1, Saga 840, Japan
}

Received January 30, 1990

We have already reported the isolation and identification of two compounds, aucuparin (1) and eriobofuran (2), from the diseased shoot and leaves of loquat (Eriobotrya japonica: Rosaceae) as its phytoalexins (Fig.1) ${ }^{1,2)}$ In our continuing research into phytoalexins of the Rosaceae plants, we respectively isolated two antifungal principles, 4'-methoxyaucuparin (3) and rhaphiolepsin (4), from an extract of mechanically wounded leaves treated with $\mathrm{HgCl}_{2}$ and from naturally fungusinfected leaves of Rhaphiolepsis umbellata (the Japanese name, "sharinbai"), which were not detected in an extract from healthy leaves. In this paper, we describe the structural elucidation of these two phytoalexins and their antifungal activities.

An EtOH extract of the mechanically wounded leaves of

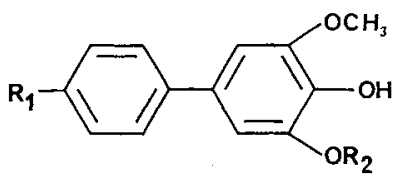

$1 \mathrm{R}_{1}=\mathrm{H}, \mathrm{R}_{2}=\mathrm{CH}_{3}$

3 $\mathrm{R}_{1}=\mathrm{OCH}_{3}, \mathrm{R}_{2}=\mathrm{CH}_{3}$

$4 \mathrm{R}_{1}=\mathrm{OCH}_{3}, \mathrm{R}_{2}=\mathrm{H}$

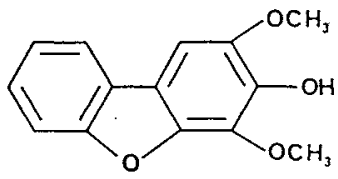

$\underline{2}$

Fig. 1. Structures of the Phytoalexins from two Rosaceae Plants.
R. umbellata treated with $10^{-3} \mathrm{M} \mathrm{HgCl}_{2}$ was purified by repeated preparative silica gel thin layer-chromatography (hexane-ethyl acetate-methanol, 60:40:1 (v/v)) with monitoring of the antifungal activity to yieid compound 3. Compound 3 exhibited the following spectroscopic properties: HRMS $m / z^{+} 260.1058\left(\mathrm{C}_{15} \mathrm{H}_{16} \mathrm{O}_{4}\right.$, requires 260.1048); MS $m / z 260\left[\mathrm{M}^{+}\right], 245\left[\mathrm{M}^{+}-\mathrm{CH}_{3}\right]$ and 149 (base peak); UV $\lambda_{\max }^{\mathrm{EtOH}} 268 \mathrm{~nm}(\varepsilon, 13,500) ;{ }^{1} \mathrm{H}-\mathrm{NMR} \delta_{\mathrm{TMS}}^{\mathrm{CDCl}_{3}}$ $7.45(2 \mathrm{H}, \mathrm{d}, J=9 \mathrm{~Hz}), 6.95(2 \mathrm{H}, \mathrm{d}, J=9 \mathrm{~Hz}), 6.76(2 \mathrm{H}, \mathrm{s})$, $5.40(1 \mathrm{H}$, br. s, $\mathrm{OH}), 3.98\left(3 \mathrm{H} \times 2, \mathrm{~s}, \mathrm{OCH}_{3}\right)$ and $3.85(3 \mathrm{H}$, $\mathrm{s}, \mathrm{OCH}_{3}$ ). Among these signals, three singlet signals at $\delta$ $6.76,5.40$ and 3.98 which were also observed in the ${ }^{1} \mathrm{H}$ NMR spectrum of aucuparin (1), suggested that one benzene ring in 3 had two methoxy groups at C-3 and C-5, and one hydroxyl function at C-4, as was the case with $\mathbf{1}$. $A$ pair of $A B$ doublet signals at $\delta 6.95$ and 7.45 indicated that another benzene ring was para-disubstituted. Upon irradiation of the doublet at $\delta 6.95$, the sharp singlet at $\delta$ 3.85 gave an NOE enhancement, suggesting that a methoxyl group stood on C-4'. Therefore, the structure of compound 3 was deduced to be 3,5,4'-trimethoxy-4hydroxybiphenyl and was designated $4^{\prime}$-methoxyaucuparin as shown in Fig. 1.

Rhaphiolepsin (4) was isolated from an EtOH extract of the leaves naturally infected with a pathogenic fungus, Entomosporium mespili, by preparative silica gel thin layer-chromatography (hexane-ethyl acetate-methanol, $60: 40: 1(\mathrm{v} / \mathrm{v}))$. This compound exhibited the following spectroscopic properties: HRMS $m / z \quad \mathrm{M}^{+} 246.0893$ $\left(\mathrm{C}_{14} \mathrm{H}_{14} \mathrm{O}_{4}\right.$, requires 246.0891$)$; MS $m / z 246\left[\mathrm{M}^{+}\right.$, base peak], and $231\left[\mathrm{M}^{+}-\mathrm{CH}_{3}\right] ; \mathrm{UV} \lambda_{\max }^{\mathrm{EtOH}} 265 \mathrm{~nm}(\varepsilon, 13,800)$;

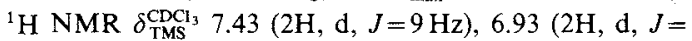
$9 \mathrm{~Hz}), 6.80(1 \mathrm{H}, \mathrm{d}, J=2 \mathrm{~Hz}), 6.63(1 \mathrm{H}, \mathrm{d}, J=2 \mathrm{~Hz}), 5.50$ (1H, br.s, OH), $5.45\left(1 \mathrm{H}\right.$, br.s, OH) $3.95\left(3 \mathrm{H}, \mathrm{s}, \mathrm{OCH}_{3}\right)$

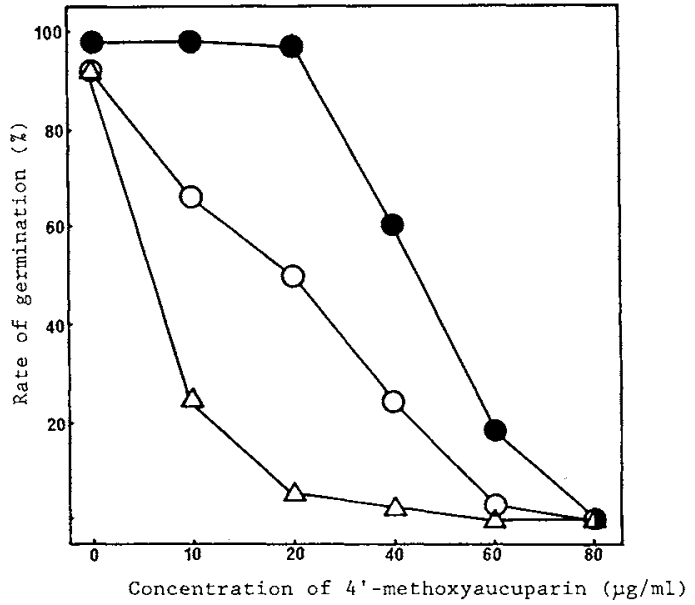

Fig. 2. Inhibitory Effect of 4'-Methoxyaucuparin (3) on the Spore Germinations of Three Fungi.

-O-, Pestalotia sp. (pathogenic); - - - M. fructicola (non-pathogenic); $-\triangle-, B$. cinerea (non-pathogenic). 


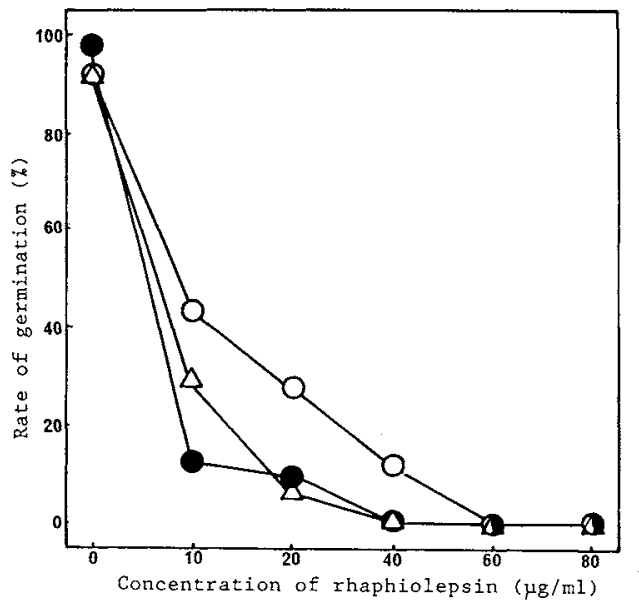

Fig. 3. Inhibitory Effect of Rhaphiolepsin (4) on the Spore Germination of Three Fungi.

The notation is the same as that described in Fig. 2.

and $3.85\left(3 \mathrm{H}, \mathrm{s}, \mathrm{OCH}_{3}\right)$. Upon saturation of the singlet at $\delta 3.85$, a doublet at $\delta 6.93$ coupled with the doublet at $\delta$ 7.43 revealed an NOE enhancement, suggesting that one benzene ring in 4 had one methoxy group at $C-4^{\prime}$ as with $4^{\prime}$-methoxyaucuparin (3). A pair of doublets at $\delta 6.63$ and 6.80 suggested that another benzene ring was not symmetrically substituted as with 1 and 3 . Upon irradiation of another methoxy signal at $\delta 3.95$, the doublet at $\delta 6.63$ gave an NOE enhancement, accounting for the presence of one methoxy group at C-3 and two hydroxy functions at C-4 and C-5, respectively. The dimethylated derivative of 4 was identical with the monomethylated derivative of 3 .
Accordingly, the structure of rhaphiolepsin (4) was deduced to be 3,4'-dimethoxy-4,5-dihydroxybiphenyl.

As is shown in Figs. 2 and 3, 4'-methoxyaucuparin (3) inhibited spore germinations of the non-pathogenic fungi, Monilinia fructicola and Botrytis cinerea, more strongly than that of a pathogenic fungus, Pestalotia sp. On the other hand, rhaphiolepsin (4) isolated from the infected leaves exhibited significant inhibiting activity against Pestalotia sp. as well as the two others, suggesting that the presence of the free hydroxyl group at C-5 of 4 contributed to its non-selective inhibitory effect on spore germination. The demethylation at C-4 of 3 seems to be carried out by a fungus, $E$. mespili, because it is known that pisatin, a phytoalexin from Pisum sativum, was demethylated at C-3 by pathogenic fungi such as Nectria haematococca. ${ }^{3)}$ Since Pestalotia sp. is phytopathogenic to R. umbellata, the growth of its hypha would be resistant to rhaphiolepsin (4), although 4 inhibits the spore germination of Pestalotia sp. The mechanism for the accumulation of these two compounds in $R$. umbellata is interesting in view of the interaction between the host plant and its phytopathogenic fungi. The detailed biological activities of both compounds are now under investigation and will be reported elsewhere in the near future.

\section{References}

1) K. Watanabe, Y. Ishiguri, F. Nonaka and A. Morita, Agric. Biol. Chem., 46, 567 (1982).

2) M. Miyakado, K. Watanabe, N. Ohno, F. Nonaka and A. Morita, J. Pesticide Sci., 10, 101 (1985).

3) H. D. Van Etten, P. S. Matthews, K. J. Tegtmeier, M. F. Dietert and J. I. Stein, Physiol. Plant Pathol., 16, 257 (1980). 